\title{
Blocking sets of tangent and external lines to an elliptic quadric in $\operatorname{PG}(3, q)$
}

\author{
BART DE BRUYN ${ }^{1}$, PUSPENDU PRADHAN ${ }^{2,3, *}$ \\ and BINOD KUMAR SAHOO ${ }^{2,3}$ \\ ${ }^{1}$ Department of Mathematics: Algebra and Geometry, Ghent University, \\ Krijgslaan 281 (S25), 9000 Gent, Belgium \\ ${ }^{2}$ School of Mathematical Sciences, National Institute of Science Education \\ and Research (NISER), Bhubaneswar, Jatni, Khurda, Odisha 752050, India \\ ${ }^{3}$ Homi Bhabha National Institute (HBNI), Training School Complex, \\ Anushakti Nagar, Mumbai 400094, India \\ *Corresponding Author \\ E-mail: Bart.DeBruyn@Ugent.be; puspendu.pradhan@niser.ac.in; \\ bksahoo@niser.ac.in
}

MS received 18 April 2020; revised 24 August 2020; accepted 30 December 2020

\begin{abstract}
Consider an elliptic quadric $Q^{-}(3, q)$ in $\operatorname{PG}(3, q)$. Let $\mathcal{E}$ and $\mathcal{T}$ denote the set of all lines of $\operatorname{PG}(3, q)$ which meet $Q^{-}(3, q)$ in 0 and 1 point, respectively. In this paper, we characterize the minimum size $(\mathcal{T} \cup \mathcal{E})$-blocking sets and give a different proof for the characterization of minimum size $\mathcal{E}$-blocking sets in $\operatorname{PG}(3, q)$ which works for all $q$. We also discuss whether the main results of this paper (Theorems 1.6 and 1.7) can be extended to ovoids in $\mathrm{PG}(3, q)$.
\end{abstract}

Keywords. Projective space; blocking set; irreducible conic; elliptic quadric; ovoid.

2010 Mathematics Subject Classification. 51E21, 05B25.

\section{Introduction}

Throughout the paper, $q$ is a prime power and $\operatorname{PG}(d, q)$ is the $d$-dimensional projective space over the finite field $\mathbb{F}_{q}$ of order $q$. For a given nonempty set $\mathcal{L}$ of lines of $\operatorname{PG}(d, q)$, a set $X$ of points of $\operatorname{PG}(d, q)$ is called an $\mathcal{L}$-blocking set if each line in $\mathcal{L}$ contains at least one point of $X$. Blocking sets in $\operatorname{PG}(d, q)$ with respect to varying sets of lines are combinatorial objects in finite geometry with several applications and have been the subject of investigation by several authors. The first step in this regard has been to determine the minimum size of an $\mathcal{L}$-blocking set and if possible, to give geometric descriptions of all $\mathcal{L}$-blocking sets of that minimum cardinality. An $\mathcal{L}$-blocking set $X$ is said to be minimal if no proper subset of $X$ is an $\mathcal{L}$-blocking set in $\operatorname{PG}(d, q)$. Clearly, every minimum size $\mathcal{L}$-blocking set in $\operatorname{PG}(d, q)$ is also a minimal $\mathcal{L}$-blocking set.

When $\mathcal{L}$ is the set of all lines of $\operatorname{PG}(d, q)$, the following fundamental result was proved by Bose and Burton [7, Theorem 1]. 


\section{PROPOSITION 1.1 [7]}

If $\mathcal{L}$ is the set of all lines of $\operatorname{PG}(d, q)$ and $X$ is an $\mathcal{L}$-blocking set in $\operatorname{PG}(d, q)$, then $|X| \geq \frac{q^{d}-1}{q-1}$, and equality holds if and only if $X$ is a hyperplane of $\operatorname{PG}(d, q)$.

\subsection{Irreducible conics in $\mathrm{PG}(2, q)$}

Let $\mathcal{C}$ be an irreducible conic in $\mathrm{PG}(2, q)$. We refer to [13] for the following basic properties of points and lines of $\operatorname{PG}(2, q)$ with respect to $\mathcal{C}$. The conic $\mathcal{C}$ consists of $q+1$ points meeting every line of $\operatorname{PG}(2, q)$ in at most two points. A line of $\operatorname{PG}(2, q)$ is called external (respectively, tangent, secant) with respect to $\mathcal{C}$ if it meets $\mathcal{C}$ in 0 (respectively, 1,2) points. Every point of $\mathcal{C}$ is contained in a unique tangent line, this gives exactly $q+1$ tangent lines to $\mathcal{C}$. There are $\frac{1}{2} q(q+1)$ secant lines and so $\left(q^{2}+q+1\right)-(q+1)-\frac{1}{2} q(q+1)=\frac{1}{2} q(q-1)$ external lines to $\mathcal{C}$. Every point of $\mathcal{C}$ is contained in $q$ secant lines.

Suppose that $q$ is even. Then all the $q+1$ tangent lines meet in a common point and this common point of intersection is called the nucleus of $\mathcal{C}$. Every point of $\mathrm{PG}(2, q) \backslash \mathcal{C}$ different from the nucleus lies on $q / 2$ secant lines, $q / 2$ external lines and a unique tangent line.

Suppose that $q$ is odd. Then every point of $\mathrm{PG}(2, q) \backslash \mathcal{C}$ lies on 0 or 2 tangent lines. Such a point is called interior to $\mathcal{C}$ in the first case and exterior to $\mathcal{C}$ in the latter. There are $\frac{1}{2} q(q-1)$ interior points and $\frac{1}{2} q(q+1)$ exterior points with respect to $\mathcal{C}$. Every interior point lies on $\frac{1}{2}(q+1)$ external lines and $\frac{1}{2}(q+1)$ secant lines. Every exterior point lies on $\frac{1}{2}(q-1)$ external lines and $\frac{1}{2}(q-1)$ secant lines. Every external line contains $\frac{1}{2}(q+1)$ interior points and $\frac{1}{2}(q+1)$ exterior points. Every secant line contains $\frac{1}{2}(q-1)$ interior points and $\frac{1}{2}(q-1)$ exterior points.

\subsection{Blocking sets in $\mathrm{PG}(2, q)$}

The minimum size $\mathcal{L}$-blocking sets in $\operatorname{PG}(2, q)$, where the line set $\mathcal{L}$ is any of the possible combinations of the sets of external, tangent and secant lines with respect to an irreducible conic $\mathcal{C}$, have been characterized by the contributions of several authors. One can refer to [15] for a brief survey of the results obtained in this regard. The following result was proved in [1, Theorem 1.2] for $q$ even and in [2, Theorem 1.1] for $q$ odd.

\section{PROPOSITION $1.2[1,2]$}

Let $\mathcal{C}$ be an irreducible conic in $\mathrm{PG}(2, q)$ and let $A$ be a blocking set of the tangent and external lines in $\mathrm{PG}(2, q)$ with respect to $\mathcal{C}$. Then $|A| \geq q$, and equality holds if and only if one of the following three cases occurs:

(i) $A=L \backslash \mathcal{C}$ for some line $L$ of $\mathrm{PG}(2, q)$ which is tangent to $\mathcal{C}$;

(ii) $A=(L \backslash \mathcal{C}) \cup\{n\}$ for some line $L$ of $\mathrm{PG}(2, q)$ which is secant to $\mathcal{C}$, where $n$ is the pole of $L$ if $q$ is odd and the nucleus of $\mathcal{C}$ if $q$ is even;

(iii) $q$ is a square and $A=\Pi \backslash(\Pi \cap \mathcal{C})$, where $\Pi$ is a Baer subplane of $\mathrm{PG}(2, q)$ such that $\Pi \cap \mathcal{C}$ is an irreducible conic in $\Pi$. 
The examples mentioned in (i) and (ii) are always blocking sets (of the tangent and external lines of $\operatorname{PG}(2, q)$ with respect to $\mathcal{C})$, while this is not always the case for the examples mentioned in (iii). In (iii), it is therefore implicitly assumed that the Baer subplane $\Pi$ needs to be chosen in such a way that $A=\Pi \backslash(\Pi \cap \mathcal{C})$ is a blocking set, and this is always possible. We refer to Sect. 2, in particular to Remark 2.2, for a further discussion of this issue. The following is an immediate consequence of Proposition 1.2.

\section{COROLLARY 1.3}

Let $\mathcal{C}$ be an irreducible conic in $\mathrm{PG}(2, q)$ and let $A$ be a blocking set of the tangent and external lines in $\mathrm{PG}(2, q)$ with respect to $\mathcal{C}$. If $|A|=q$, then $A \cap \mathcal{C}=\emptyset$.

We refer to [3, Theorem 1.1] for $q$ odd and to [11, Theorem 1.1] for $q$ even for a proof of the following proposition.

\section{PROPOSITION $1.4[3,11]$}

Let $\mathcal{C}$ be an irreducible conic in $\mathrm{PG}(2, q)$ and let $A$ be a blocking set in $\mathrm{PG}(2, q)$ of the external lines to $\mathcal{C}$. Then $|A| \geq q-1$.

The following result was proved in [17, Lemma 2.4], also see [6, Proposition 3.1].

\section{PROPOSITION $1.5[6,17]$}

Let $x$ be a point of $\mathrm{PG}(2, q)$ and let $\mathcal{L}$ be the set of all lines of $\mathrm{PG}(2, q)$ not containing $x$. If $A$ is an $\mathcal{L}$-blocking set in $\mathrm{PG}(2, q)$, then $|A| \geq q$ and equality holds if and only if $A=L \backslash\{x\}$ for some line L through $x$.

\subsection{Elliptic quadrics in $\mathrm{PG}(3, q)$}

Let $Q^{-}(3, q)$ be an elliptic quadric in $\mathrm{PG}(3, q)$, that is, a nondegenerate quadric in $\mathrm{PG}(3, q)$ of Witt index one. We refer to [12] for the basic properties of points, lines and planes of PG $(3, q)$ with respect to $Q^{-}(3, q)$. The quadric $Q^{-}(3, q)$ consists of $q^{2}+1$ points and it meets every line in at most two points. Accordingly, the lines of $\operatorname{PG}(3, q)$ are divided into three collections:

(i) the set $\mathcal{E}$ of external lines which are disjoint from $Q^{-}(3, q)$,

(ii) the set $\mathcal{T}$ of tangent lines which meet $Q^{-}(3, q)$ in a singleton,

(iii) the set $\mathcal{S}$ of secant lines which meet $Q^{-}(3, q)$ in two points.

Every point of $Q^{-}(3, q)$ is contained in $q+1$ tangent lines, this gives $|\mathcal{T}|=(q+1)\left(q^{2}+1\right)$. We also have $|\mathcal{S}|=\frac{1}{2} q^{2}\left(q^{2}+1\right)$ and then $|\mathcal{E}|=\left(q^{2}+1\right)\left(q^{2}+q+1\right)-\frac{1}{2} q^{2}\left(q^{2}+1\right)-$ $(q+1)\left(q^{2}+1\right)=\frac{1}{2} q^{2}\left(q^{2}+1\right)$.

With the quadric $Q^{-}(3, q)$, there is naturally associated a polarity $\tau$ which is symplectic if $q$ is even, and orthogonal if $q$ is odd. Thus $\tau$ is an inclusion reversing bijection of order two on the set of all subspaces of $\mathrm{PG}(3, q)$, which fixes the line set of $\mathrm{PG}(3, q)$, and interchanges the point set and the set of all planes of PG(3,q). For every point $x$ of $Q^{-}(3, q)$, the plane $x^{\tau}$ of $\mathrm{PG}(3, q)$ intersects $Q^{-}(3, q)$ at the point $x$ and the $q+1$ tangent 
lines through $x$ are precisely the lines through $x$ contained in $x^{\tau}$. In this case, $x^{\tau}$ is called a tangent plane with tangency point $x$. For every point $x$ of $\mathrm{PG}(3, q) \backslash Q^{-}(3, q)$, the plane $x^{\tau}$ of $\operatorname{PG}(3, q)$ is called a secant plane and the intersection $\mathcal{C}_{x^{\tau}}:=x^{\tau} \cap Q^{-}(3, q)$ is an irreducible conic in $x^{\tau}$. The map $x \mapsto \mathcal{C}_{x^{\tau}}$ defines a bijection between $\mathrm{PG}(3, q) \backslash Q^{-}(3, q)$ and the set of conics contained in $Q^{-}(3, q)$.

Suppose that $q$ is even. Then, for every point $x$ of $\operatorname{PG}(3, q) \backslash Q^{-}(3, q)$, the secant plane $x^{\tau}$ contains the point $x$ and the tangent lines contained in $x^{\tau}$ are precisely the $q+1$ lines of $x^{\tau}$ through $x$. Thus the point $x$ is the nucleus of the conic $\mathcal{C}_{x} \tau$ in $x^{\tau}$.

Suppose that $q$ is odd. Then, for every point $x$ of $\operatorname{PG}(3, q) \backslash Q^{-}(3, q)$, the secant plane $x^{\tau}$ does not contain the point $x$ and the tangent lines through $x$ are precisely the $q+1$ lines through $x$ meeting the conic $\mathcal{C}_{x^{\tau}}$ in $x^{\tau}$.

Every point of $Q^{-}(3, q)$ is contained in $q^{2}$ secant lines but no external lines. Every point of $\mathrm{PG}(3, q) \backslash Q^{-}(3, q)$ is contained in $\frac{1}{2} q(q-1)$ secant lines and $\frac{1}{2} q(q+1)$ external lines. There are $q^{2}+1$ tangent planes and $q^{3}+q$ secant planes. Every tangent line is contained in one tangent plane and $q$ secant planes. Every secant line is contained in $q+1$ secant planes. Every external line is contained in two tangent planes and $q-1$ secant planes. Every point of $Q^{-}(3, q)$ is contained in one tangent plane and $q^{2}+q$ secant planes. Every point of $\mathrm{PG}(3, q) \backslash Q^{-}(3, q)$ is contained in $q+1$ tangent planes and $q^{2}$ secant planes.

\subsection{Blocking sets in $\mathrm{PG}(3, q)$}

Recently, we have characterized in [8] the minimum size $\mathcal{L}$-blocking sets in $\operatorname{PG}(3, q)$, where $\mathcal{L}$ is one of the line sets $\mathcal{S}, \mathcal{E} \cup \mathcal{S}$ and $\mathcal{T} \cup \mathcal{S}$. In this paper, we prove the following result:

Theorem 1.6. Let $B$ be a $(\mathcal{T} \cup \mathcal{E})$-blocking set in $\mathrm{PG}(3, q)$. Then $|B| \geq q^{2}+q$, and equality holds if and only if $B=x^{\tau} \backslash\{x\}$ for some point $x$ of $Q^{-}(3, q)$.

In [6, Section 3], Biondi et al. studied $\mathcal{E}$-blocking sets in $\operatorname{PG}(3, q)$ and characterized such blocking sets of minimum size for $q \geq 9$. In this paper, we give an alternate proof characterizing the minimum size $\mathcal{E}$-blocking sets in $\operatorname{PG}(3, q)$ which works for all $q$. More precisely, the following result holds for $\mathcal{E}$-blocking sets:

Theorem 1.7. Let $B$ be an $\mathcal{E}$-blocking set in $\mathrm{PG}(3, q)$. Then $|B| \geq q^{2}$, and equality holds if and only if $B=\pi \backslash Q^{-}(3, q)$ for some secant plane $\pi$ of $\mathrm{PG}(3, q)$.

In Sect. 2, we prove a result concerning blocking sets of the tangent and external lines in $\operatorname{PG}(2, q)$ with respect to an irreducible conic in $\mathrm{PG}(2, q)$. Theorems 1.6 and 1.7 are proved in Sects. 3 and 4, respectively. Finally, in Sect. 5, we discuss whether Theorems 1.6 and 1.7 can be extended to ovoids in $\mathrm{PG}(3, q)$.

We note that the minimum size blocking sets in $\mathrm{PG}(3, q)$ of similar line sets with respect to a hyperbolic quadric have been characterized in the papers $[5,6,9,10,16-18]$.

\section{A result in $\operatorname{PG}(2, q)$}

We shall need the following result in $\mathrm{PG}(2, q)$ while proving Theorem 1.6 for odd $q$. 
Lemma 2.1. Let $\mathcal{C}$ be an irreducible conic in $\mathrm{PG}(2, q), q$ square, and let $\Pi$ be a Baer subplane of $\mathrm{PG}(2, q)$ such that $\Pi \cap \mathcal{C}$ is an irreducible conic in $\Pi$. Suppose that $A:=$ $\Pi \backslash(\Pi \cap \mathcal{C})$ is a blocking set of the tangent and external lines in $\mathrm{PG}(2, q)$ with respect to $\mathcal{C}$. Then the following hold for a line $L$ of $\mathrm{PG}(2, q)$ that is tangent to $\mathcal{C}$ with tangency point $x$ :

(i) If $x \in \Pi \cap \mathcal{C}$, then L intersects $\Pi$ in a Baer subline that is tangent to $\Pi \cap \mathcal{C}$ in the point $x$.

(ii) If $x \in \mathcal{C} \backslash \Pi$, then L cannot intersect $\Pi$ in a Baer subline.

Proof.

(i) Suppose $x \in \Pi \cap \mathcal{C}$. Then as $L$ meets $A=\Pi \backslash(\Pi \cap \mathcal{C})$, it must intersect $\Pi$ in a Baer subline and this Baer subline necessarily coincides with the line of $\Pi$ that is tangent to $\Pi \cap \mathcal{C}$ in the point $x$.

(ii) Suppose $x \in \mathcal{C} \backslash \Pi$ and $q$ is even. Let $y_{1}$ and $y_{2}$ be two distinct points of $\Pi \cap \mathcal{C}$. Let $L_{i}$ with $i \in\{1,2\}$ denote the unique line of $\operatorname{PG}(2, q)$ that is tangent to $\mathcal{C}$ in the point $y_{i}$. By (i), we know that $L_{1} \cap \Pi$ and $L_{2} \cap \Pi$ are two distinct lines of $\Pi$ that are tangent to $\Pi \cap \mathcal{C}$. So, $L_{1} \cap \Pi$ and $L_{2} \cap \Pi$ meet in the nucleus $n$ of the conic $\mathcal{C} \cap \Pi$ in $\Pi$. As $n \in L_{1} \cap L_{2}$, the point $n$ is also the nucleus of $\mathcal{C}$. Now closedup, the line $L$ must contain the nucleus $n$. If $L \cap \Pi$ were a Baer subline, then $L \cap \Pi$ would be a line of $\Pi$ that is tangent to $\mathcal{C} \cap \Pi$ in a point $y$. As $y \in L \cap \mathcal{C}$, we have $x=y$, in contradiction with the fact that $x \notin \Pi$.

Suppose $x \in \mathcal{C} \backslash \Pi$ and $q$ is odd. We can choose a reference system in $\operatorname{PG}(2, q)$ with respect to which $\Pi$ consists of all points $\left(X_{1}, X_{2}, X_{3}\right)$ of $\mathrm{PG}(2, q)$ with $X_{1}, X_{2}, X_{3} \in$ $\mathbb{F}_{\sqrt{q}}$ and $\Pi \cap \mathcal{C}$ consists of all points $\left(X_{1}, X_{2}, X_{3}\right)$ of $\operatorname{PG}(2, q)$ with $X_{1}, X_{2}, X_{3} \in \mathbb{F}_{\sqrt{q}}$ satisfying $X_{1}^{2}+X_{2} X_{3}=0$. Suppose $\mathcal{C}$ has equation $\sum_{1 \leq i \leq j \leq 3} a_{i j} X_{i} X_{j}=0$ with respect to the same reference system, where $a_{i j} \in \mathbb{F}_{q}$ for all $i, j \in\{1,2,3\}$ with $i \leq j$. Since $(1,1,-1),(1,-1,1),(0,1,0)$ and $(0,0,1)$ belong to $\mathcal{C} \cap \Pi \subseteq \mathcal{C}$, the equation of $\mathcal{C}$ is of the form $a_{11}\left(X_{1}^{2}+X_{2} X_{3}\right)+a_{12}\left(X_{1} X_{2}+X_{1} X_{3}\right)=0$, that is, of the form $X_{1}^{2}+X_{2} X_{3}+b\left(X_{1} X_{2}+X_{1} X_{3}\right)=0$ for some $b \in \mathbb{F}_{q} \backslash\{1,-1\}$ as $\mathcal{C}$ is irreducible. The line $\bar{K}$ of $\operatorname{PG}(2, q)$ that is tangent to $\mathcal{C}$ in the point $(0,1,0)$ has equation $X_{3}+b X_{1}=0$, while the line $K$ of $\Pi$ that is tangent to $\Pi \cap \mathcal{C}$ in the point $(0,1,0)$ has equation $X_{3}=0$. By (i), we know that $\bar{K} \cap \Pi=K$. This implies that $b=0$, that is, $\mathcal{C}$ has also equation $X_{1}^{2}+X_{2} X_{3}=0$ with respect to the same reference system (but coordinates are in $\mathbb{F}_{q}$ ). Now, suppose that $x$ has coordinates $\left(u_{1}, u_{2}, u_{3}\right)$ and that $L$ intersects $\Pi$ in a Baer subline. The line $L$ has equation $a_{1} X_{1}+a_{2} X_{2}+a_{3} X_{3}=0$, where

$$
\left[\begin{array}{l}
a_{1} \\
a_{2} \\
a_{3}
\end{array}\right]:=\left[\begin{array}{lll}
2 & 0 & 0 \\
0 & 0 & 1 \\
0 & 1 & 0
\end{array}\right] \cdot\left[\begin{array}{l}
u_{1} \\
u_{2} \\
u_{3}
\end{array}\right] .
$$

Since $L \cap \Pi$ is a Baer subline, $\left(a_{1}, a_{2}, a_{3}\right)$ is proportional to a nonzero element of $\mathbb{F}^{3} \sqrt{q}$. This implies by (1) that $\left(u_{1}, u_{2}, u_{3}\right)$ is also proportional to a nonzero element of $\mathbb{F}^{3} \sqrt{q}$. As this is in contradiction with the fact that $x \in \mathcal{C} \backslash \Pi$, our assumption that $L$ intersects $\Pi$ in a Baer subline was wrong.

Remark 2.2. If $\mathcal{C}$ is an irreducible conic in $\operatorname{PG}(2, q), q$ square, and $\Pi$ is a Baer subplane of PG $(2, q)$ intersecting $\mathcal{C}$ in an irreducible conic of $\Pi$, then for $q \geq 16, A:=\Pi \backslash(\Pi \cap \mathcal{C})$ is always a blocking set of the tangent and external lines of $\operatorname{PG}(2, q)$ with respect to $\mathcal{C}$, while counter examples to that claim exist for $q=4$ and $q=9$. 
We justify Remark 2.2 in the following. As in the proof of Lemma 2.1, we can take a reference system in $\mathrm{PG}(2, q)$ with respect to which $\Pi$ consists of all points $\left(X_{1}, X_{2}, X_{3}\right)$ of $\operatorname{PG}(2, q)$ with $X_{1}, X_{2}, X_{3} \in \mathbb{F}_{\sqrt{q}}$ and $\Pi \cap \mathcal{C}$ consists of all points $\left(X_{1}, X_{2}, X_{3}\right)$ of $\operatorname{PG}(2, q)$ with $X_{1}, X_{2}, X_{3} \in \mathbb{F} \sqrt{q}$ satisfying $X_{1}^{2}+X_{2} X_{3}=0$. Suppose $\mathcal{C}$ has equation $\sum_{1 \leq i \leq j \leq 3} a_{i j} X_{i} X_{j}=0$ with respect to the same reference system. The fact that the points $(0,1,0),(0,0,1)$ and $\left(1, \omega,-\omega^{-1}\right)$ belong to $\mathcal{C}$ for every $\omega \in \mathbb{F} \sqrt{q} \backslash\{0\}$ then implies that $a_{22}=a_{33}=0$ and $a_{12} \omega^{2}+\left(a_{11}-a_{23}\right) \omega-a_{13}=0$ for all $\omega \in \mathbb{F} \sqrt{q} \backslash\{0\}$. For $q \geq 16$, this implies that $a_{12}=a_{13}=0, a_{11}=a_{23}$ and so $\mathcal{C}$ also has equation $X_{1}^{2}+X_{2} X_{3}=0$ with respect to the same reference system (another way to see this is as follows: For $q \geq 16$, we have $|\Pi \cap \mathcal{C}|=\sqrt{q}+1 \geq 5$. As there is a unique irreducible conic through any given collection of five points of which no three are on the same line, we thus see that $\mathcal{C}$ coincides with the irreducible conic with equation $X_{1}^{2}+X_{2} X_{3}=0$ ). It is now straightforward to verify that for every point $x \in \Pi \cap \mathcal{C}$, we have $K_{x}=\overline{K_{x}} \cap \Pi$, where $\overline{K_{x}}$ and $K_{x}$ are the tangent lines of $\operatorname{PG}(2, q)$ and $\Pi$ through $x$ with respect to $\mathcal{C}$ and $\Pi \cap \mathcal{C}$, respectively. This property implies that $A=\Pi \backslash(\Pi \cap \mathcal{C})$ is a blocking set of the tangent and external lines of $\operatorname{PG}(2, q)$ with respect to $\mathcal{C}$.

For $q \in\{4,9\}$, it is possible that $\mathcal{C}$ has equation $X_{1}^{2}+X_{2} X_{3}+\omega\left(X_{1} X_{2}+X_{1} X_{3}\right)=0$ with $\omega \in \mathbb{F}_{q} \backslash \mathbb{F}_{\sqrt{q}}$. We can then verify that $K \neq \bar{K} \cap \Pi$, where $\bar{K}$ and $K$ are the tangent lines of $\mathrm{PG}(2, q)$ and $\Pi$ through $(0,1,0)$ with respect to $\mathcal{C}$ and $\Pi \cap \mathcal{C}$, respectively. The line $\bar{K}$ does not contain any points of $A=\Pi \backslash(\Pi \cap \mathcal{C})$ and so $A$ cannot be a blocking set of the tangent and external lines with respect to $\mathcal{C}$.

\section{3. $(\mathcal{T} \cup \mathcal{E})$-blocking sets}

\subsection{Basic properties}

Let $\pi$ be a plane of $\operatorname{PG}(3, q)$. The lines of $\mathcal{T} \cup \mathcal{E}$ that are contained in $\pi$ will be denoted by $(\mathcal{T} \cup \mathcal{E})_{\pi}$. If $\pi$ is a secant plane, then $(\mathcal{T} \cup \mathcal{E})_{\pi}$ is the set of all tangent and external lines of $\pi$ with respect to the conic $\mathcal{C}_{\pi}:=\pi \cap Q^{-}(3, q)$ in $\pi$. If $\pi$ is a tangent plane, then it does not contain any line of $\operatorname{PG}(3, q)$ that is secant to $Q^{-}(3, q)$ and so $(\mathcal{T} \cup \mathcal{E})_{\pi}$ is the set of all lines of $\pi$. In both cases, for a $(\mathcal{T} \cup \mathcal{E})$-blocking set $B$ in $\operatorname{PG}(3, q)$, the set $B_{\pi}:=\pi \cap B$ is a $(\mathcal{T} \cup \mathcal{E})_{\pi}$-blocking set in $\pi$. If $\pi$ is a secant plane, then $\left|B_{\pi}\right| \geq q$ by Proposition 1.2. If $\pi$ is a tangent plane, then Proposition 1.1 (with $d=2$ ) implies that $\left|B_{\pi}\right| \geq q+1$ and equality holds if and only if $B_{\pi}$ is a line of $\pi$. We thus have the following.

Lemma 3.1. Let $B$ be a $(\mathcal{T} \cup \mathcal{E})$-blocking set in $\mathrm{PG}(3, q)$ and $\pi$ be a plane of $\mathrm{PG}(3, q)$. Then the following hold:

(i) If $\pi$ is a secant plane, then $\left|B_{\pi}\right| \geq q$.

(ii) If $\pi$ is a tangent plane, then $\left|B_{\pi}\right| \geq q+1$ and equality holds if and only if $B_{\pi}$ is a line of $\pi$.

Now let $B$ be a $(\mathcal{T} \cup \mathcal{E})$-blocking set in $\operatorname{PG}(3, q)$ of minimum cardinality. For any point $x$ of $Q^{-}(3, q)$, it is clear that the point set $x^{\tau} \backslash\{x\}$ blocks every line of $\mathcal{T} \cup \mathcal{E}$ and so is a $(\mathcal{T} \cup \mathcal{E})$-blocking set in $\operatorname{PG}(3, q)$ of size $q^{2}+q$. Then minimality of $|B|$ implies that $|B| \leq q^{2}+q$. 
Lemma 3.2. The following hold:

(i) There exists a secant line of $\mathrm{PG}(3, q)$ that is disjoint from $B$. If $L$ is such a secant line, then each of the $q+1$ (secant) planes through $L$ contains exactly $q$ points of $B$.

(ii) $|B|=q^{2}+q$ and $B \cap Q^{-}(3, q)=\emptyset$.

Proof. Since $|B| \leq q^{2}+q$, Proposition 1.1 implies that there exists a secant line $L$ of $\operatorname{PG}(3, q)$ that is disjoint from $B$. Each plane through $L$, being a secant plane, contains at least $q$ points of $B$ by Lemma 3.1(i). Since $L$ is disjoint from $B$, the $q+1$ secant planes through $L$ together contain at least $q^{2}+q$ points of $B$. It follows that $|B|=q^{2}+q$ and that each secant plane through $L$ contains exactly $q$ points of $B$.

For a given secant plane $\pi$ through $L,\left|B_{\pi}\right|=q$ implies that $B_{\pi}$ is disjoint from the conic $\mathcal{C}_{\pi}$ in $\pi$ by Corollary 1.3. Since $B$ is a disjoint union of the sets $B_{\pi}$, and $Q^{-}(3, q)$ is the union of the conics $\mathcal{C}_{\pi}$ as $\pi$ runs over all the secant planes through $L$, it follows that $B$ is disjoint from $Q^{-}(3, q)$.

\subsection{Properties of tangent planes}

Lemma 3.3. If $\pi$ is a tangent plane with $\left|B_{\pi}\right|=q+1$, then $B_{\pi}$ is an external line.

Proof. By Lemma 3.1(ii), $B_{\pi}$ is a line of $\pi$. Since $B$ is disjoint from $Q^{-}(3, q)$ by Lemma 3.2(ii), it follows that $B_{\pi}$ is an external line.

Lemma 3.4. There are at least two tangent planes each containing $q+1$ points of $B$.

Proof. By Lemma 3.1(ii), every tangent plane contains at least $q+1$ points of $B$. Let $a$ be the number of tangent planes containing exactly $q+1$ points of $B$. Consider the set

$$
Y=\{(x, \pi): x \in B, \pi \text { is a tangent plane containing } x\} .
$$

Every point of $\mathrm{PG}(3, q) \backslash Q^{-}(3, q)$ is contained in $q+1$ tangent planes. Using Lemma 3.2(ii), we count $|Y|$ in two ways to get

$$
\left(q^{2}+q\right)(q+1)=|B|(q+1)=|Y| \geq a(q+1)+\left(q^{2}+1-a\right)(q+2) .
$$

This gives $a \geq 2$.

Lemma 3.5. If $\pi_{1}$ and $\pi_{2}$ are two distinct tangent planes with $\left|B_{\pi_{1}}\right|=q+1=\left|B_{\pi_{2}}\right|$, then the external lines $B_{\pi_{1}}$ and $B_{\pi_{2}}$ are either equal or meet in a point.

Proof. The external line $\pi_{1} \cap \pi_{2}$ of $\pi_{2}$ contains a point of the line $B_{\pi_{2}}$. This point of $B$ lies in $\pi_{1}$ and hence is contained in $B_{\pi_{1}}$. So, the lines $B_{\pi_{1}}$ and $B_{\pi_{2}}$ meet.

Lemma 3.6. For every external line $L$, there are at most two tangent planes $x^{\tau}, x \in$ $Q^{-}(3, q)$, such that $B_{x^{\tau}}=L$.

Proof. This follows from the fact that there are precisely two tangent planes through $L$ (see Section 1.3). 
Lemma 3.7. Suppose $\pi$ is a tangent plane with tangency point $x$. Then the number of secant lines through $x$ disjoint from $B$ is at least $\left|B_{\pi}\right|-q \geq 1$.

Proof. By Lemma 3.1(ii), $\left|B_{\pi}\right| \geq q+1$ and so $\left|B_{\pi}\right|-q \geq 1$. We have $x \notin B$ and $|B|=q^{2}+q$ by Lemma 3.2(ii). There are $|B|-\left|B_{\pi}\right|=q^{2}-\left(\left|B_{\pi}\right|-q\right)$ points of $B \backslash \pi$ and these are distributed over the $q^{2}$ (secant) lines through $x$ not contained in $\pi$. So, at least $\left|B_{\pi}\right|-q$ of these $q^{2}$ lines are disjoint from $B$.

\subsection{Proof of Theorem 1.6 for $q$ odd}

Lemma 3.8. Suppose that $q$ is odd. Then every tangent line meets $B$ in either $1, \sqrt{q}$ or $q$ points.

Proof. Consider a tangent line $L$ with $L \cap Q^{-}(3, q)=\{x\}$. By Lemma 3.7, there exists a secant line $K$ through $x$ disjoint from $B$. Put $\pi:=\langle K, L\rangle$, the secant plane generated by $K$ and $L$. By Lemma 3.2(i), $\pi$ intersects $B$ in precisely $q$ points. Since $B_{\pi}$ is a $(\mathcal{T} \cup \mathcal{E})_{\pi^{-}}$ blocking set in $\pi$ of size $q$, one of the following three possibilities occurs for $B_{\pi}$ by Proposition 1.2.

(1) Suppose that $B_{\pi}=M \backslash \mathcal{C}_{\pi}$ for some line $M$ of $\pi$ tangent to $\mathcal{C}_{\pi}$. In $\pi$, if $L=M$, then $|L \cap B|=q$, and if $L \neq M$, then $|L \cap B|=1$ as $L$ and $M$ intersect in a point not in $\mathcal{C}_{\pi}$

(2) Suppose that $B_{\pi}=\left(M \backslash \mathcal{C}_{\pi}\right) \cup\{n\}$ for some line $M$ of $\pi$ secant to $\mathcal{C}_{\pi}$, where $n$ is the pole of $M$ with respect to $\mathcal{C}_{\pi}$. If $x \in M \cap \mathcal{C}_{\pi}$, then $L$ passes through the pole $n$ of $M$ and so $|L \cap B|=1$. If $x \notin M \cap \mathcal{C}_{\pi}$, then $L$ does not pass through $n$ and meets $M \backslash \mathcal{C}_{\pi}$ in a point, implying $|L \cap B|=1$.

(3) Suppose that $q$ is square and $B_{\pi}=\mu \backslash\left(\mu \cap \mathcal{C}_{\pi}\right)$, where $\mu$ is a Baer subplane of $\pi$ such that $\mu \cap \mathcal{C}_{\pi}$ is an irreducible conic in $\mu$. Put $K \cap Q^{-}(3, q)=\{x, y\}$. Note that every line of $\pi$ meets $\mu$ in a singleton or a set of $\sqrt{q}+1$ points. Since $K$ is disjoint from $B$, it intersects $\mu$ in a unique point, and this point must belong to $\mathcal{C}_{\pi}$. So, one of $x, y$ belongs to $\mu \cap \mathcal{C}_{\pi}$ while the other belongs to $\mathcal{C}_{\pi} \backslash \mu$. If $x \in \mu \cap \mathcal{C}_{\pi}$ and $y \in \mathcal{C}_{\pi} \backslash \mu$, then $L$ intersects $B$ in $\sqrt{q}$ points by Lemma 2.1(i). Assume therefore that $y \in \mu \cap \mathcal{C}_{\pi}$ and $x \in \mathcal{C}_{\pi} \backslash \mu$. Then Lemma 2.1(ii) implies that the tangent line $L$ of $\pi$ through $x$ meets $\mu$ in a unique point not belonging to $\mathcal{C}_{\pi}$, that is, $|L \cap B|=1$.

Remark 3.9. The proof of Lemma 3.8 does not work for $q$ even. Otherwise, in Step (2), we then have that $B_{\pi}=\left(M \backslash \mathcal{C}_{\pi}\right) \cup\{n\}$, where $n$ is the nucleus of $\mathcal{C}_{\pi}$. In the case that $x \notin M \cap \mathcal{C}-\pi$, we then have that $L$ contains two points of $B$, namely the nucleus $n$ and a point of $M \backslash \mathcal{C}_{\pi}$.

For every $i \in\{1, \sqrt{q}, q\}$, we denote by $N_{i}$ the number of tangent lines meeting $B$ in exactly $i$ points.

Lemma 3.10. Suppose that $q$ is odd. Then the following hold:

(i) $N_{1} \geq(q+1)\left(q^{2}-\sqrt{q}\right)$ and $N_{\sqrt{q}}+N_{q} \leq(\sqrt{q}+1)(q+1)$. 
(ii) If $N_{\sqrt{q}}=0$, then $N_{1}=q^{2}(q+1)$ and $N_{q}=q+1$.

Proof. Since there are $q+1$ tangent lines through each point of $Q^{-}(3, q)$, we see that

$$
N_{1}+N_{\sqrt{q}}+N_{q}=\left|Q^{-}(3, q)\right| \cdot(q+1)=\left(q^{2}+1\right)(q+1) .
$$

Consider the set $Z=\{(b, L): b \in B, \quad L$ is a tangent line containing $b\}$. By Lemma 3.2(ii), $|B|=q^{2}+q$ and $B \subseteq \mathrm{PG}(3, q) \backslash Q^{-}(3, q)$. Recall also that each point of $\operatorname{PG}(3, q) \backslash Q^{-}(3, q)$ is contained in exactly $q+1$ tangent lines. Counting $|Z|$ in two ways, we have

$$
N_{1}+\sqrt{q} \cdot N_{\sqrt{q}}+q \cdot N_{q}=|B| \cdot(q+1)=\left(q^{2}+q\right)(q+1) .
$$

If $N_{\sqrt{q}}=0$, then (2) and (3) imply that $N_{1}=q^{2}(q+1)$ and $N_{q}=q+1$. This proves (ii). In the general case, we deduce from (2) and (3) that

$$
(\sqrt{q}-1) N_{1}-(q-\sqrt{q}) N_{q}=(q+1) \cdot\left[\sqrt{q}\left(q^{2}+1\right)-\left(q^{2}+q\right)\right] .
$$

So,

$$
(\sqrt{q}-1) N_{1} \geq(q+1) \cdot\left[(\sqrt{q}-1) q^{2}-\sqrt{q}(\sqrt{q}-1)\right],
$$

that is, $N_{1} \geq(q+1)\left(q^{2}-\sqrt{q}\right)$. Then (2) implies that $N_{\sqrt{q}}+N_{q} \leq(\sqrt{q}+1)(q+1)$. This proves (i).

Let $\Gamma$ denote the set of all points $x$ of $Q^{-}(3, q)$ such that the tangent plane $x^{\tau}$ meets $B$ in exactly $q+1$ points. By Lemma $3.4,|\Gamma| \geq 2$. This bound is improved in the following lemma for odd $q$.

Lemma 3.11. If $q$ is odd, then $|\Gamma| \geq 2 q>q+1$.

Proof. For $x \in \Gamma, B_{x^{\tau}}$ is an external line by Lemma 3.3 and so every tangent line through $x$ meets $B$ in a singleton. If $q$ is not a square, then $N_{\sqrt{q}}=0$ and so $N_{q}=q+1$ by Lemma 3.10(ii). This implies that there are at most $q+1$ points of $Q^{-}(3, q)$ which are not contained in $\Gamma$. So,

$$
|\Gamma| \geq\left(q^{2}+1\right)-(q+1)=q^{2}-q \geq 2 q,
$$

where the last inequality holds as $q \geq 3$ for $q$ being odd.

If $q$ is a square, then $N_{\sqrt{q}}+N_{q} \leq(q+1)(\sqrt{q}+1)$ by Lemma 3.10(i). This implies that at most $(q+1)(\sqrt{q}+1)$ points of $Q^{-}(3, q)$ are not contained in $\Gamma$. So,

$$
|\Gamma| \geq\left(q^{2}+1\right)-(q+1)(\sqrt{q}+1) \geq 2 q,
$$

where the second inequality holds as $q \geq 9$ for $q$ being an odd square.

\section{PROPOSITION 3.12}

If $q$ is odd, then $B$ is equal to the point set of a tangent plane minus its tangency point in $Q^{-}(3, q)$. 
Proof. Recall that, for $x \in \Gamma$, the tangent plane $x^{\tau}$ meets $B$ in the external line $L_{x}:=B_{x^{\tau}}$. The collection $L_{x}, x \in \Gamma$, of external lines mutually intersect by Lemma 3.5. There are two possibilities: all these lines contain a given point or are contained in a given plane.

Suppose first that all the lines of the collection $L_{x}, x \in \Gamma$, share a common point, say $u$. Then $u \in L_{x} \subseteq x^{\tau}$ for every $x \in \Gamma$. So, $x \in u^{\tau}$ for every $x \in \Gamma$, implying that the plane $u^{\tau}$ intersects $Q^{-}(3, q)$ in at least $|\Gamma|>q+1$ points (Lemma 3.11), a contradiction.

Therefore, the external lines $L_{x}, x \in \Gamma$, are all contained in the same plane, say $\mu^{*}$. For every such external line $L$, there are at most two points $x \in \Gamma$ such that $L_{x}=L$ by Lemma 3.6. This fact and Lemma 3.11 imply that the number of mutually distinct lines in the collection $L_{x}, x \in \Gamma$, is at least $\frac{|\Gamma|}{2} \geq q$. Let $L_{1}, L_{2}, \ldots, L_{q}$ be $q$ mutually distinct lines of this collection. We then have

$$
\begin{aligned}
\left|B \cap \mu^{*}\right| \geq & \left|L_{1}\right|+\left|L_{2} \backslash L_{1}\right|+\left|L_{3} \backslash\left(L_{1} \cup L_{2}\right)\right| \\
& +\cdots+\left|L_{q} \backslash\left(L_{1} \cup L_{2} \cup \cdots \cup L_{q-1}\right)\right| \\
\geq & (q+1)+q+\cdots+2=\frac{q(q+3)}{2} .
\end{aligned}
$$

We claim that $\mu^{*} \backslash Q^{-}(3, q)$ is contained in $B$. Suppose there exists a point $u$ of $\mu^{*} \backslash\left(Q^{-}(3, q) \cup B\right)$. There are $q+1$ tangent lines and $\frac{q^{2}+q}{2}$ external lines of $\operatorname{PG}(3, q)$ through $u$. So, there are at least $\frac{q^{2}+q}{2}$ tangent or external lines through $u$ which are not contained in $\mu^{*}$. Each of these lines contains a point of $B \backslash \mu^{*}$. So,

$$
|B|=\left|B \cap \mu^{*}\right|+\left|B \backslash \mu^{*}\right| \geq \frac{q(q+3)}{2}+\frac{q^{2}+q}{2}=q^{2}+2 q,
$$

in contradiction with the fact that $|B|=q^{2}+q$.

Thus $\mu^{*} \backslash Q^{-}(3, q) \subseteq B$. If $\mu^{*}$ is a tangent plane, then $|B|=q^{2}+q$ implies that $B$ is equal to the point set of the tangent plane $\mu^{*}$ minus its tangency point. In order to complete the proof of the proposition, we now show that $\mu^{*}$ cannot be a secant plane. If $\mu^{*}$ is a secant plane, then $B_{\mu^{*}}=\mu^{*} \backslash \mathcal{C}_{\mu *}=\mu^{*} \backslash Q^{-}(3, q)$ contains $q^{2}$ points. Since $|B|=q^{2}+q$, we get

$$
\left|B \backslash \mu^{*}\right|=q \geq 2 .
$$

In fact, for every $x \in \mathcal{C}_{\mu *}$, each of the $q$ tangent lines through $x$ not in $\mu^{*}$ contains a unique point of $B \backslash \mu^{*}$. This implies that $y \in x^{\tau}$ for every $x \in \mathcal{C}_{\mu *}$ and every $y \in B \backslash \mu^{*}$. This is impossible as the intersection $\bigcap_{x \in \mathcal{C}_{\mu *}} x^{\tau}$ is a singleton, namely $\left(\mu^{*}\right)^{\tau}$.

\subsection{Proof of Theorem 1.6 for $q$ even}

Lemma 3.13. Suppose that $q$ is even. Let $x$ be a point of $\mathrm{PG}(3, q) \backslash Q^{-}(3, q)$. If $x$ is not a point of $B$, then $\left|B_{x^{\tau}}\right| \geq q+1$.

Proof. Since $q$ is even, every line of $x^{\tau}$ through $x$ is a tangent line. Each such line must contain a point of $B$, that is, a point of $B_{x^{\tau}}$. Since $x \notin B$, it follows that $\left|B_{x} \tau\right| \geq q+1$.

As a consequence of Lemma 3.13, we have 


\section{COROLLARY 3.14}

Suppose that $q$ is even. Let $x$ be a point of $\mathrm{PG}(3, q) \backslash Q^{-}(3, q)$. If the secant plane $x^{\tau}$ contains exactly $q$ points of $B$, then $x \in B$.

Lemma 3.15. Suppose that $q$ is even. Then the following hold for points $x$ of $\mathrm{PG}(3, q) \backslash$ $Q^{-}(3, q)$ :

(i) $x \in B$ if and only if $\left|B_{x^{\tau}}\right|=q$.

(ii) $x \notin B$ if and only if $\left|B_{x} \tau\right|=q+1$.

Proof. By Lemma 3.1(i), every secant plane of $\mathrm{PG}(3, q)$ contains at least $q$ points of $B$. Let $R$ be the collection of all points $x$ of $\mathrm{PG}(3, q) \backslash Q^{-}(3, q)$ for which $\left|B_{x^{\tau}}\right|=q$.

(i) It is enough to show that $R=B$. We have $R \subseteq B$ by Corollary 3.14, and so $|R| \leq$ $|B|=q^{2}+q$. Consider the following set

$$
X=\{(b, \pi): b \in B, \pi \text { is a secant plane containing } b\} .
$$

The number of secant planes of $\mathrm{PG}(3, q)$ is $q^{3}+q$ and each point of $\mathrm{PG}(3, q) \backslash Q^{-}(3, q)$ is contained in exactly $q^{2}$ secant planes. Counting $|X|$ in two ways using Lemma 3.2(ii), we get

$$
\left(q^{2}+q\right) q^{2}=|B| \cdot q^{2}=|X| \geq|R| \cdot q+\left(q^{3}+q-|R|\right)(q+1) .
$$

This gives $|R| \geq q^{2}+q$. Thus $|R|=q^{2}+q=|B|$ and hence $R=B$.

(ii) Let $s$ denote the number of secant planes of $\mathrm{PG}(3, q)$ containing exactly $q+1$ points of $B$. Using (i), we have $s \leq q^{3}+q-|B|=q^{3}+q-\left(q^{2}+q\right)=q^{3}-q^{2}$. Consider again the set $X$ defined in the proof of (i) above. We count $|X|$ in two ways to get

$$
\left(q^{2}+q\right) q^{2}=|X| \geq\left(q^{2}+q\right) q+s(q+1)+\left(q^{3}+q-\left(q^{2}+q\right)-s\right)(q+2) .
$$

This gives $s \geq q^{3}-q^{2}$. Thus $s=q^{3}-q^{2}$ and it follows that each of the $q^{3}-q^{2}$ secant planes $x^{\tau}$ with $x \notin B$ meets $B$ in exactly $q+1$ points.

\section{PROPOSITION 3.16}

If $q$ is even, then $B$ is equal to the point set of a tangent plane minus its tangency point in $Q^{-}(3, q)$.

Proof. Let $\pi_{0}$ be a tangent plane containing exactly $q+1$ points of $B$. Such a plane $\pi_{0}$ exists by Lemma 3.4. Then $B_{\pi_{0}}$ is an external line by Lemma 3.3 and so it is contained in two tangent planes and $q-1$ secant planes. Let $\pi_{1}$ be the other tangent plane and $\pi_{2}, \ldots, \pi_{q}$ be the secant planes through $B_{\pi_{0}}$. Then $B_{\pi_{i}}$ contains $B_{\pi_{0}}$ for every $i$. By Lemma 3.15, every secant plane contains $q$ or $q+1$ points of $B$. This implies that $B_{\pi_{j}}=B_{\pi_{0}}$ for $2 \leq j \leq q$. Since $B=\bigcup_{i=0}^{q} B_{\pi_{i}}$, it now follows that $B=B_{\pi_{1}}$. Since $B$ is disjoint from $Q^{-}(3, q)$ and $|B|=q^{2}+q$ by Lemma 3.2(ii), we must have that $B=B_{\pi_{1}}=\pi_{1} \backslash\{y\}$, where $y$ is the tangency point of $\pi_{1}$ in $Q^{-}(3, q)$. 


\section{Minimum size $\mathcal{E}$-blocking sets}

We start by noting that Theorem 1.7 was proved by Biondi et al. in [6, Theorem 3.5], with exception of the equality case for some small values of $q$, namely $q \in\{2,3,4,5,7,8\}$. We observed that their proof also works for all even $q$, but not for $q=3,5,7$. By Proposition 1.4, the minimum size of a blocking set of the external lines with respect to an irreducible conic in $\mathrm{PG}(2, q)$ is $q-1$. For $q=3,5,7$, there are examples of blocking sets of size $q-1$ of the external lines with respect to an irreducible conic in $\operatorname{PG}(2, q)$ consisting of interior points (see [9, Theorem 2.1]) and the argument used to prove [6, Theorem 3.5] does not cover such sporadic examples in the plane case.

In the rest of this section, our aim is to give an alternate proof of the equality case in Theorem 1.7 which works for all $q$, in particular, for $q=3,5,7$. We implicitly borrow some of the arguments used in [6, Section 3] for our proof.

Let $B$ be an $\mathcal{E}$-blocking set in $\operatorname{PG}(3, q)$ of minimum size. If $\pi$ is a secant plane, then $\pi \backslash Q^{-}(3, q)$ is an $\mathcal{E}$-blocking set of size $q^{2}$. From the minimality of $|B|$ and the fact that $B \backslash Q^{-}(3, q)$ is an $\mathcal{E}$-blocking set, we have that $|B| \leq q^{2}$ and that $B \cap Q^{-}(3, q)$ is empty.

For every plane $\pi$, we put $B_{\pi}:=B \cap \pi$ and denote by $\mathcal{E}_{\pi}$ the set of lines of $\mathcal{E}$ contained in $\pi$. Then $B_{\pi}$ is an $\mathcal{E}_{\pi}$-blocking set in $\pi$. Note that if $\pi$ is a tangent plane, then $\left|B_{\pi}\right| \geq q$ by Proposition 1.5.

A proof of the following lemma can easily be extracted from [6, Proposition 3.4]. We add a proof here for reasons of completeness.

Lemma 4.1. Let $N_{1}$ denote the number of tangent planes intersecting $B$ in exactly $q$ points. Then $N_{1} \geq q+1$. If $N_{1}=q+1$, then $|B|=q^{2}$ and each of the remaining $q^{2}-q$ tangent planes meets $B$ in exactly $q+1$ points.

Proof. There are $q^{2}+1-N_{1}$ tangent planes intersecting $B$ in at least $q+1$ points by Proposition 1.5 . As each point of $\mathrm{PG}(3, q) \backslash Q^{-}(3, q)$ is contained in exactly $q+1$ tangent planes, a double counting of the incident point-tangent plane pairs $(x, \pi)$ with $x \in B$ yields

$$
N_{1} \cdot q+\left(q^{2}+1-N_{1}\right) \cdot(q+1) \leq|B| \cdot(q+1) \leq q^{2}(q+1),
$$

that is, $q+1 \leq N_{1}$. If $N_{1}=q+1$, then the above implies that $|B|=q^{2}$ and that each of the $q^{2}+1-N_{1}=q^{2}-q$ tangent planes that meets $B$ in at least $q+1$ points meet it in precisely $q+1$ points.

In the sequel, let $\pi_{1}, \pi_{2}, \ldots, \pi_{k}$ with $k \geq q+1$ be all the tangent planes intersecting $B$ in precisely $q$ points. Let $\alpha_{i}$ with $i \in\{1,2, \ldots, k\}$ be the tangency point of $\pi_{i}$. By proposition 1.5, there exists a line $U_{i}$ of $\pi_{i}$ through $\alpha_{i}$ such that $B \cap \pi_{i}=U_{i} \backslash\left\{\alpha_{i}\right\}$.

Lemma 4.2. Any two distinct lines in the collection $\left\{U_{1}, U_{2}, \ldots, U_{k}\right\}$ intersect in a singleton (outside $Q^{-}(3, q)$ ).

Proof. Let $i_{1}, i_{2} \in\{1,2, \ldots, k\}$ with $i_{1} \neq i_{2}$. The external line $L=\pi_{i_{1}} \cap \pi_{i_{2}}$ intersects $B$ in a singleton $\{\beta\}$ which belongs to both $U_{i_{1}}$ and $U_{i_{2}}$. Hence $U_{i_{1}}=\alpha_{i_{1}} \beta$ and $U_{i_{2}}=\alpha_{i_{2}} \beta$ meet in the singleton $\{\beta\}$. 
Lemma 4.3. We have $k=q+1$ and $U_{1}, U_{2}, \ldots, U_{q+1}$ are the $q+1$ tangent lines contained in a secant plane $\pi^{*}$.

Proof. As $\left\{U_{1}, U_{2}, \ldots, U_{k}\right\}$ is a collection of mutually intersecting lines by Lemma 4.2, at least one of the following cases occurs:

(1) the lines $U_{1}, U_{2}, \ldots, U_{k}$ are contained in the same plane $\pi^{*}$;

(2) the lines $U_{1}, U_{2}, \ldots, U_{k}$ go through the same point $x^{*}$.

We show that Case (1) occurs. Note that if Case (2) occurs, then $x^{*} \notin Q^{-}(3, q)$ by Lemma 4.2.

If Case (2) occurs with $q$ even, then the tangent lines $U_{1}, U_{2}, \ldots, U_{k}$ are all contained in the secant plane $\left(x^{*}\right)^{\tau}$ through $x^{*}$, indeed showing Case (1) occurs.

Suppose that Case (2) occurs with $q$ odd. Then the tangent lines through $x^{*}$ are the $q+1$ lines through $x^{*}$ containing a point of $\left(x^{*}\right)^{\tau} \cap Q^{-}(3, q)$. As $k \geq q+1$, we then have $k=q+1$. As $\left|B \cap\left(U_{1} \cup U_{2} \cup \ldots \cup U_{q+1}\right)\right|=1+(q+1)(q-1)=q^{2}$ and $|B| \leq q^{2}$, we must have $B=\left(U_{1} \cup U_{2} \cup \ldots \cup U_{q+1}\right) \backslash Q^{-}(3, q)$. But this is impossible, as any external line contained in the secant plane $\left(x^{*}\right)^{\tau}$ would then be disjoint from $B$.

Thus, Case (1) occurs. As the $k \geq q+1$ tangent lines $U_{1}, U_{2}, \ldots, U_{k}$ are all contained in the same plane and their tangency points are mutually distinct, we can conclude that $k=q+1, \pi^{*}$ is a secant plane and that $U_{1}, U_{2}, \ldots, U_{q+1}$ are all the $q+1$ tangent lines contained in $\pi^{*}$.

The following is a consequence of Lemmas 4.1 and 4.3.

\section{COROLLARY 4.4}

We have $|B|=q^{2}$. There are $q+1$ tangent planes meeting $B$ in precisely $q$ points and $q^{2}-q$ tangent planes meeting $B$ in precisely $q+1$ points.

The following lemma completes the proof of Theorem 1.7.

Lemma 4.5. Let $\pi^{*}$ be the secant plane as in Lemma 4.3. Then $B=\pi^{*} \backslash Q^{-}(3, q)$.

Proof. For $q$ odd, let $E_{\pi^{*}}$ and $I_{\pi^{*}}$ denote the set of points of $\pi^{*}$ that are exterior and interior, respectively, with respect to the conic $\mathcal{C}_{\pi^{*}}$. Lemma 4.3 implies that the set of points of $B$ covered by the tangent lines contained in $\pi^{*}$ is precisely $\pi^{*} \backslash Q^{-}(3, q)$ if $q$ is even, and $E_{\pi^{*}}$ if $q$ is odd. Since $|B|=q^{2}=\left|\pi^{*} \backslash Q^{-}(3, q)\right|$, the lemma follows for even $q$ and it suffices to prove that $I_{\pi^{*}} \subseteq B$ for odd $q$.

Suppose to the contrary that there exists a point $x \in I_{\pi^{*}}$ which is not contained in $B$. There are $\frac{q^{2}+q}{2}-\frac{q+1}{2}=\frac{q^{2}-1}{2}$ external lines through $x$ which are not contained in $\pi^{*}$. Each of these $\frac{q^{2}-1}{2}$ external lines contains at least one point of $B \backslash \pi^{*}$, implying that

$$
\begin{aligned}
|B| & =\left|B \cap \pi^{*}\right|+\left|B \backslash \pi^{*}\right| \\
& \geq\left|E_{\pi^{*}}\right|+\frac{q^{2}-1}{2}=\frac{q^{2}+q}{2}+\frac{q^{2}-1}{2}=q^{2}+\frac{q-1}{2},
\end{aligned}
$$

in contradiction with $|B|=q^{2}$. 


\section{Generalizations to ovoids in $\operatorname{PG}(3, q)$}

An oval in the projective plane $\mathrm{PG}(2, q)$ is a set of $q+1$ points no three of which are collinear. A famous theorem of Segre [19] states that every oval in $\mathrm{PG}(2, q), q$ odd, is an irreducible conic. The same is true for $q \in\{2,4\}$. But PG(2, $q)$, with $q \geq 8$ even, has ovals that are not irreducible conics. If $O$ is an oval and $L$ is a line of $\operatorname{PG}(2, q)$, then $|L \cap O| \leq 2$. We say that $L$ is an external, tangent or secant line depending on whether $|L \cap O|$ is equal to 0,1 or 2 . All the basic properties of points and lines with respect to an irreducible conic that are mentioned in Section 1.1 remain valid with respect to an oval also. In particular, if $O$ is an oval in $\operatorname{PG}(2, q), q$ even, then there exists a unique point $n$ in $\mathrm{PG}(2, q) \backslash O$ that is contained in all the $q+1$ tangent lines. This point $n$ is called the nucleus of $O$.

An ovoid in $\mathrm{PG}(3, q)$ is a set of $q^{2}+1$ points intersecting each plane in either a singleton or an oval. Every elliptic quadric in $\mathrm{PG}(3, q)$ is an example of an ovoid. In fact, if $q$ is odd, then every ovoid is also an elliptic quadric [4,14], but more examples exist for $q$ even. If $\mathcal{O}$ is an ovoid and $L$ is a line of $\operatorname{PG}(3, q)$, then $|L \cap \mathcal{O}| \leq 2$. We say that $L$ is an external, tangent or secant line depending on whether $|L \cap \mathcal{O}|$ is equal to 0,1 or 2 .

One can now wonder whether the main results of the present paper (Theorems 1.6 and 1.7) extend to ovoids in $\mathrm{PG}(3, q)$. Since every ovoid in $\mathrm{PG}(3, q), q$ odd, is an elliptic quadric, we may restrict ourselves to the case where $q$ is even. Below, we mention some known properties of ovoids in $\mathrm{PG}(3, q), q$ even. We refer to [12] for more background information on such ovoids.

Let $\mathcal{O}$ be an ovoid in $\operatorname{PG}(3, q), q$ even. Then associated with $\mathcal{O}$, there is a symplectic polarity $\tau$ [20]. Planes intersecting $\mathcal{O}$ in a singleton are called tangent planes while planes intersecting $\mathcal{O}$ in ovals are called secant planes.

If $x \in \mathcal{O}$, then $x^{\tau}$ is a tangent plane and every other plane through $x$ is a secant plane. Through $x$, there are $q+1$ tangent lines (namely the $q+1$ lines of $x^{\tau}$ through $x$ ) and $q^{2}$ secant lines.

If $x \in \mathrm{PG}(3, q) \backslash \mathcal{O}$, then $x^{\tau}$ is a secant plane intersecting $\mathcal{O}$ in $q+1$ points, say $y_{1}, y_{2}, \ldots, y_{q+1}$. The planes $y_{1}^{\tau}, y_{2}^{\tau}, \ldots, y_{q+1}^{\tau}$ are the $q+1$ tangent planes through $x$, while the remaining $q^{2}$ planes through $x$ are secant planes. The point $x$ is contained in precisely $q+1$ tangent lines (namely $\left.x y_{1}, x y_{2}, \ldots, x y_{q+1}\right), \frac{q^{2}-q}{2}$ secant lines and $\frac{q^{2}+q}{2}$ external lines.

Also, every external line with respect to $\mathcal{O}$ is contained in two tangent planes and $q-1$ secant planes.

We thus observe that a general ovoid in $\mathrm{PG}(3, q), q$ even, satisfies similar properties as an elliptic quadric. This raises the hope that Theorems 1.6 and 1.7 can be generalized to arbitrary ovoids. The main obstacle in doing so is however that the proofs of these theorems rely on Corollary 1.3, Proposition 1.4 and the inequality $|A| \geq q$ from Proposition 1.2. These results were proved in the literature for irreducible conics. In the following proposition, we extend these results to arbitrary ovals.

\section{PROPOSITION 5.1}

Let $O$ be an oval in $\mathrm{PG}(2, q), q$ even, and let $n$ be the nucleus of $O$. Then the following hold:

(i) If $A$ is a blocking set in $\mathrm{PG}(2, q)$ with respect to the external lines to $O$, then $|A| \geq$ $q-1$. If $|A|=q-1$, then $A \cap(O \cup\{n\})=\emptyset$. 
(ii) If $A$ is a blocking set in $\mathrm{PG}(2, q)$ with respect to the external and tangent lines to $O$, then $|A| \geq q$.

(iii) The point sets of size $q$ in $\mathrm{PG}(2, q)$ that block all tangent and external lines to $O$ are precisely the sets of the form $A \cup\{n\}$, where $A$ is a blocking set of size $q-1$ with respect to the external lines to $O$. In particular, such sets of points are always disjoint from $O$.

Proof.

(i) It suffices to prove this claim for minimal blocking sets $A$ with respect to the external lines to $O$. Since $A \backslash(O \cup\{n\})$ is also a blocking set in $\mathrm{PG}(2, q)$ with respect to the external lines to $O$, minimality of $A$ implies that $A \cap(O \cup\{n\})=\emptyset$. So, every point of $A$ is contained in $\frac{q}{2}$ external lines. As there are $\frac{q(q-1)}{2}$ external lines each containing at least one point of $A$, the number of points in $A$ is at least $\frac{q(q-1)}{2}\left(\frac{q}{2}\right)^{-1}=q-1$. Hence, $|A| \geq q-1$.

If $|A|=q-1$, then $A$ necessarily is a minimal blocking set and by the above, we then know that $A \cap(O \cup\{n\})=\emptyset$.

(ii) Suppose that $|A| \leq q-1$. As $A$ is also a blocking set with respect to the external lines to $O$, we know from (i) that $|A|=q-1$ and $A \cap(O \cup\{n\})=\emptyset$. Each point of $A$ is therefore contained in $\frac{q}{2}+1=\frac{q+2}{2}$ lines that are external or tangent to $O$. In total, there are $\frac{q(q-1)}{2}+q+1=\frac{q^{2}+q+2}{2}$ lines in $\operatorname{PG}(2, q)$ that are external or tangent to $O$. As each such line contains at least one point of $A$, we have $|A| \geq \frac{q^{2}+q+2}{2} \cdot\left(\frac{q+2}{2}\right)^{-1}>q-1$, a contradiction.

(iii) If $A$ is a blocking set of size $q-1$ with respect to the external lines to $O$, then $A \cup\{n\}$ is a blocking set of size $q$ with respect to the external and tangent lines to $O$ (as each tangent line contains $n$ ). Conversely, suppose $A^{\prime}$ is a blocking set of size $q$ with respect to the external and tangent lines to $O$. If $n \notin A^{\prime}$, then each of the $q+1$ lines through $n$ is a tangent line containing a point of $A^{\prime}$, proving that $\left|A^{\prime}\right| \geq q+1$, a contradiction. Hence, $n \in A^{\prime}$. Obviously, $A:=A^{\prime} \backslash\{n\}$ is a blocking set of size $q-1$ with respect to the external lines to $O$. By (i), we know that $A$ is disjoint from $O$. Hence, also $A^{\prime}=A \cup\{n\}$ is then disjoint from $O$.

Taking into account Proposition 5.1 and the above-mentioned properties of ovoids in $\mathrm{PG}(3, q), q$ even, we can now easily verify that the arguments mentioned in Sects. 3.1, 3.2, 3.4 and 4 remain valid for general ovoids. So, the conclusions of Theorems 1.6 and 1.7 remain valid for general ovoids in $\mathrm{PG}(3, q), q$ even. We thus have the following theorems:

Theorem 5.2. Let $\mathcal{O}$ be an ovoid in $\mathrm{PG}(3, q)$ and let $B$ be a blocking set of the external lines in $\mathrm{PG}(3, q)$ with respect to $\mathcal{O}$. Then $|B| \geq q^{2}$, and equality holds if and only if $B=\pi \backslash \mathcal{O}$ for some secant plane $\pi$ of $\mathrm{PG}(3, q)$ with respect to $\mathcal{O}$.

Theorem 5.3. Let $\mathcal{O}$ be an ovoid in $\mathrm{PG}(3, q)$ and let $B$ be a blocking set of the external and tangent lines in $\mathrm{PG}(3, q)$ with respect to $\mathcal{O}$. Then $|B| \geq q^{2}+q$, and equality holds if and only if $B=\pi \backslash \mathcal{O}$ for some tangent plane $\pi$ of $\mathrm{PG}(3, q)$ with respect to $\mathcal{O}$.

\section{Acknowledgements}

The first author would like to thank the National Institute of Science Education and Research, Bhubaneswar, for the kind hospitality provided during his visit to the School 
of Mathematical Sciences in January 2020. The second author is supported by a PhD Fellowship (File No. 09/1002(0040)/2017-EMR-I) of the Council of Scientific and Industrial Research (CSIR), Ministry of Science and Technology, Government of India. The third author is partially supported by Project No. MTR/2017/000372 of the Science and Engineering Research Board (SERB), Department of Science and Technology, Government of India.

\section{References}

[1] Aguglia A and Giulietti M, Blocking sets of certain line sets related to a conic, Des. Codes Cryptogr. 39 (2006) 397-405

[2] Aguglia A and Korchmáros G, Blocking sets of nonsecant lines to a conic in $\mathrm{PG}(2, q), q$ odd, J. Combin. Des. 13 (2005) 292-301

[3] Aguglia A and Korchmáros G, Blocking sets of external lines to a conic in $\mathrm{PG}(2, q), q$ odd, Combinatorica 26 (2006) 379-394

[4] Barlotti A, Un'estensione del teorema di Segre-Kustaanheimo, Boll. Un. Mat. Ital. (3) 10 (1955) 498-506

[5] Biondi P and Lo Re P M, On blocking sets of external lines to a hyperbolic quadric in PG(3, q), $q$ even, J. Geom. 92 (2009) 23-27

[6] Biondi P, Lo Re P M and Storme L, On minimum size blocking sets of external lines to a quadric in PG(3, q), Beiträge Algebra Geom. 48 (2007) 209-215

[7] Bose R C and Burton R C, A characterization of flat spaces in a finite geometry and the uniqueness of the Hamming and the MacDonald codes, J. Combinatorial Theory 1 (1966) 96-104

[8] De Bruyn B, Pradhan P and Sahoo B K, Minimum size blocking sets of certain line sets with respect to an elliptic quadric in PG(3, q), Contrib. Discrete Math. 15(2) (2020) 132-147

[9] De Bruyn B, Sahoo B K and Sahu B, Blocking sets of tangent and external lines to a hyperbolic quadric in $\mathrm{PG}(3, q)$, Discrete Math. 341 (2018) 2820-2826

[10] De Bruyn B, Sahoo B K and Sahu B, Blocking sets of tangent lines to a hyperbolic quadric in PG(3, 3), Discrete Appl. Math. 266 (2019) 121-129

[11] Giulietti M, Blocking sets of external lines to a conic in PG $(2, q), q$ even, European J. Combin. 28 (2007) $36-42$

[12] Hirschfeld J W P, Finite Projective Spaces of Three Dimensions, Oxford Mathematical Monographs (1985) (Oxford University Press)

[13] Moorhouse G E, Incidence Geometry, 2017, available online at http://ericmoorhouse.org/ handouts/Incidence_Geometry.pdf

[14] Panella G, Caratterizzazione delle quadriche di uno spazio (tridimensionale) lineare sopra un corpo finito, Boll. Un. Mat. Ital. (3) 10 (1955) 507-513

[15] Patra K L, Sahoo B K and Sahu B, Minimum size blocking sets of certain line sets related to a conic in PG(2, q), Discrete Math. 339 (2016) 1716-1721

[16] Sahoo B K and Sahu B, Blocking sets of tangent and external lines to a hyperbolic quadric in PG (3, q), $q$ even, Proc. Indian Acad. Sci. (Math. Sci.) 129(1) (2019) Art. 4, 14 pp.

[17] Sahoo B K and Sahu B, Blocking sets of certain line sets to a hyperbolic quadric in $\operatorname{PG}(3, q)$, Adv. Geom. 19 (2019) 477-486

[18] Sahoo B K and Sastry N S N, Binary codes of the symplectic generalized quadrangle of even order, Des. Codes Cryptogr. 79 (2016) 163-170

[19] Segre B. Ovals in a finite projective plane, Canadian J. Math. 7 (1955) 414-416

[20] Segre B, On complete caps and ovaloids in three-dimensional Galois spaces of characteristic two, Acta Arith. 5 (1959) 315-332 